\title{
New Analysis on Sparse Solutions to Random Standard Quadratic Optimization Problems and Extensions
}

\author{
Xin Chen *Jiming Peng ${ }^{\dagger}$
}

April 26, 2012

\begin{abstract}
The standard quadratic optimization problem (StQP) refers to the problem of minimizing a quadratic form over the standard simplex. Such a problem arises from numerous applications and is known to be NPhard. In a recent paper [15], we showed that with a high probability close to 1 , StQPs with random data have sparse optimal solutions when the associated data matrix is randomly generated from a certain distribution such as uniform and exponential distributions. In this paper, we present a new analysis for random StQPs combining probability inequalities derived from both the first-order and second-order optimality conditions. The new analysis allows us to significantly improve the probability bounds. More important, it allows us to handle normal distributions which is left open in [15]. The existence of sparse approximate solutions to convex StQPs and extensions to other classes of QPs are discussed as well.
\end{abstract}

Key words. Co-positive definite matrix, standard quadratic programming, sparse solutions, random matrices, probability analysis.

\section{Introduction}

In this paper, we consider the following quadratic programming problem

$$
\begin{array}{cl}
\min & x^{T} Q x \\
\text { s.t. } & e^{T} x=1, \quad x \geq 0,
\end{array}
$$

${ }^{*}$ Department of Industrial and Enterprise System Engineering, University of Illinois at Urbana-Champaign. Urbana, IL, 61801. The research of this author is supported by NSF grant CMMI-1030923. Email: xinchen@illinois.edu.

${ }^{\dagger}$ Department of Industrial and Enterprise System Engineering, University of Illinois at Urbana-Champaign. Urbana, IL, 61801. Email:pengj@illinois.edu. The research of the corresponding author is jointly supported by NSF grants DMS 09-15240 ARRA and CMMI1131690 . 
where $Q=\left[Q_{i j}\right] \in \Re^{n \times n}$ is a symmetric matrix, and $e \in \Re^{n}$ is the all 1-vector. Like in [6], we called the above problem the standard quadratic programming problem (StQP). The problem appears in numerous applications such as resource allocation [26], portfolio selection [30] and machine learning [32]. It also covers several other important problems such as the maximal clique problem in discrete optimization [20] and the determination of co-positivity of a matrix in linear algebra [9]. In addition to its broad range of applications, the StQP provides a prototype for numerous classes of quadratic optimization problems. As such, the study of StQP has caught the attention of experts in many different fields and various algorithms have been proposed in the literature. For details, we refer to recent papers $[9,36,41]$ and the references therein.

In this work we are mainly concerned with a special scenario of StQPs where the matrix $Q$ is generated from a certain distribution. The study on optimization problems with random data can be dated back to early 1980s [21] when Goldberg and Marchetti-Spaccamela considered how to find an exact solution of the knapsack problem with random data. For more recent progress on random knapsack problems, we refer to the paper [4]. In recent years, the study on the so-called $L_{1}$ minimization problem with random constraints has attracted a great amount of attention from experts in various disciplines. It is shown that when the coefficient matrix is generated from a normal distribution, then with a high probability, the optimal solution of the $L_{1}$ minimization problem is the sparest point in the constrained set $[12,13,19]$. In [34], the authors extended the $L_{1}$ minimization to the case where the decision variable is required to be a positive semidefinite (PSD) matrix and the $L_{1}$ norm is replaced by the Nuclear norm. Other extensions can also be found in [14].

In our recent work [15], we noted that there exists a close relation between the StQP and the $L_{1}$ minimization. Inspired by this observation, we considered the existence of sparse solutions to StQPs with random data and proved that with a high probability, an StQP admits a very sparse optimal solution if the matrix $Q$ is generated from a class of distributions including the uniform or exponential distributions.

The analysis in [15] mainly builds upon on one characteristic based on the first-order optimality condition of the global optimal solution of an StQP and some probability bounds related to order statistics for random variables with concave cumulative distribution functions in their supports. The class of distributions, though very broad and including notable distributions such as uniform and exponential, excludes normal distributions which are predominately assumed in the literature on the $L_{1}$ minimization problem and its extensions.

The main purpose of this work is to extend and improve the results in [15]. Specifically, we develop a new analysis for StQPs with random matrices combining properties derived from both the first-order and the second-order optimality conditions of the global optimal solution of an StQP. It significantly improves the results in [15]. More importantly, coupled with results from random matrix theory, our new analysis allows us to handle StQPs with random matrices generated from normal distributions. We also analyze an StQP with a random positive semi-definite $Q$ and estimate the sparsity of its so-called $\epsilon$-approximate 
solution.

The paper is organized as follows. In Section 2, we review some useful properties to be used here. In Section 3, we present a new analysis on sparse solutions to random StQPs. In Section 4, we consider the case when $Q$ is positive semidefinite, and establish the existence of sparse approximations to convex StQPs. Finally, we conclude our paper with some remarks in Section 5.

A few sentences regarding the notation. Throughout this paper, we use $e$ and $\exp (x)$ to denote the all-ones vector and the exponential function respectively. The symbol $E$ represents the all-ones square matrix with dimensions consistent with the context and we use $E_{k}$ for $E$ when the dimension is specified as $k$. We refer to $x^{*}$ as one of the sparsest global optimal solutions of problem (1) (unless else specified), i.e., it has the smallest number of positive components among all global optimal solutions. For a given vector $x \in \Re^{n},\|x\|_{0}$ denotes the number of nonzero elements. We define $n$ ! as the factorial of a natural number $n, C(n, k)$ the number of $k$-combinations from a given set with $n$ elements and $B(n, k)$ the Beta function with $B(n, k)=\int_{0}^{1} u^{n-1}(1-u)^{k-1} d u=\frac{(n-1) !(k-1) !}{(n+k-1) !}$.

\section{Preliminary Results}

Before presenting our new analysis, we first review in this section some results established in [15] that will be used in this paper.

Let $U_{r}, r=1, \ldots, n$ be independent continuous random variables each with a cumulative distribution $F(\cdot)$ and a probability density function $f(\cdot)$ and $u_{1} \leq$ $u_{2} \leq \ldots \leq u_{n}$ be the order statistics of $U_{r}$ 's. Let $V_{r}, r=1,2, \ldots, m$ be independent continuous random variables each with a cumulative distribution $G(\cdot)$ and a probability density function $g(\cdot)$ and $v_{1} \leq v_{2} \leq \ldots \leq v_{m}$ be the order statistics of $V_{r}$ 's. Assume that the random vectors $\left[U_{r}\right]_{r=1}^{n}$ and $\left[V_{s}\right]_{s=1}^{m}$ are independent from each other. In addition, we make the following assumption on the distributions.

Assumption 2.1. (a) $G(\cdot)$ is concave on its support.

(b) There exists a constant $\alpha \in(0,1]$ such that $G(x) \geq \alpha F(x)$ for any $x \in$ $(-\infty, \infty)$.

We have the following probability bounds on the order statistics.

Theorem 2.2. ([15]) Let $U_{r}, r=1, \ldots, n$ and $V_{r}, r=1, \ldots, m$ be independently identically distributed random variables with cumulative distribution functions $F(\cdot)$ and $G(\cdot)$ respectively. Assume that the random vectors $\left[U_{r}\right]_{r=1}^{n}$ and $\left[V_{s}\right]_{s=1}^{m}$ are independent from each other. If $G(\cdot)$ and $F(\cdot)$ satisfy Assumption 2.1, then we have

$$
\begin{gathered}
P\left(\sum_{r=1}^{k} u_{r} \leq k v_{1}\right) \leq \prod_{j=1}^{k} \frac{(m+n-k)(n-j+1)}{\left(n-k+\frac{\alpha m(k-j+1)}{k}\right)(m+n-j+1)} \\
\sum_{i=1}^{m} P\left(\sum_{r=1}^{k} u_{r} \leq(k+1) v_{1}-v_{i}\right) \leq(k+1) P\left(\sum_{r=1}^{k} u_{r}<k v_{1}\right) .
\end{gathered}
$$


The following proposition presents some characteristics of a global optimal solution of the standard quadratic program (1).

Proposition 2.3. ([15]) Suppose that $x^{*}$ is one of the sparsest global optimal solutions of problem (1) satisfying $\left\|x^{*}\right\|_{0}=k>1$. Let $Q_{\mathcal{K}} \in \Re^{k \times k}$ be the principal submatrix of $Q$ induced by the index set of all the nonzero elements of $x^{*}$ and define $\lambda^{*}=\left(x^{*}\right)^{T} Q x^{*}$. Then the following conclusions hold:

C.1 There exists a row (or column) of $Q_{\mathcal{K}}$ such that the average of all its elements is strictly less than the minimal diagonal element of $Q$;

C.2 The matrix $Q_{\mathcal{K}}-\lambda^{*} E_{k}$ is positive semidefinite.

Property C.1 in the above proposition follows from the first-order optimality condition, while property C.2 is derived from the second-order optimality condition. The following sparsity results of the global optimal solution were mainly built upon property C.1 and the probability bounds in Theorem 2.2.

Theorem 2.4. ([15]) Assume that the upper triangular elements of $Q$ are independent with the diagonal elements identically distributed with $c d f G(\cdot)$ and pdf $g(\cdot)$ and the strict upper triangular elements of $Q$ identically distributed with cdf $F(\cdot)$ and pdf $f(\cdot)$. Suppose that $G(\cdot)$ and $F(\cdot)$ satisfy Assumption 2.1. Let $x^{*}$ is a global optimal solution of problem (1). Then it holds

$$
P\left(\left\|x^{*}\right\|_{0} \geq k\right) \leq \tau^{k-1}\left(\frac{1}{(1-\tau)^{2}}+\frac{k-1}{1-\tau}\right)+\frac{n(n+1)}{2} \tau^{\lfloor\sqrt{2 n \alpha}\rfloor},
$$

where $\tau=\left(\frac{1}{1+\alpha / 2}\right)^{1 / 2}$.

One significant restriction of the above result is that the $\operatorname{cdf} G(\cdot)$ is required to be concave or equivalently its pdf $g(\cdot)$ is nonincreasing in its support. Though it covers notable distributions such as uniform and exponential, it excludes normal distributions, widely used in the literature of $L_{1}$ minimization. Interestingly, as observed from the numerical experiment in [15], the StQPs generated from normal distributions still have very sparse optimal solutions. In what follows we present a new analysis employing both properties C.1 and C.2, which allows us to significantly improve the probability bounds in Theorem 2.4 and more importantly quantify the sparsity of global optimal solutions of StQPs generated from normal distributions. 


\section{A New Analysis on Sparse Solutions to ran- dom StQPs}

To present our analysis, let $Q_{\mathcal{K}}$ be the principal submatrix induced by the index set $\mathcal{K}$. Define the following probability events:

$$
\begin{array}{r}
\mathcal{H}_{1}^{\mathcal{K}}=\left\{Q_{\mathcal{K}} \text { satisfies C.1 }\right\} ; \\
\mathcal{H}_{1, i}^{\mathcal{K}}=\left\{\sum_{j \in \mathcal{K}} Q_{i j} \leq k \min _{j=1: n} Q_{j j}\right\} ; \\
\tilde{\mathcal{H}}_{1, i}^{\mathcal{K}}=\left\{\sum_{j \in \mathcal{K}} Q_{i j} \leq k \min _{j \notin \mathcal{K} \backslash\{i\}} Q_{j j}\right\} ; \\
\mathcal{H}_{2}^{\mathcal{K}}=\left\{\exists \lambda^{*} \in \Re: Q_{\mathcal{K}}-\lambda^{*} E_{k} \succeq 0\right\} ; .
\end{array}
$$

Let $x^{*}$ be one of the sparsest global optimal solutions of problem (1). From Propositions 2.3, we have that if $\left\|x^{*}\right\|_{0}=k$, then there exists an index set $\mathcal{K}$ with $|\mathcal{K}|=k$ satisfying properties C.1 and C.2. Therefore, we have

$$
P\left(\left\|x^{*}\right\|_{0}=k\right) \leq C(n, k) P\left(\mathcal{H}_{1}^{\mathcal{K}} \cap \mathcal{H}_{2}^{\mathcal{K}}\right) .
$$

Because $\mathcal{H}_{1}^{\mathcal{K}}=\cup_{i \in \mathcal{K}} \mathcal{H}_{1, i}^{\mathcal{K}}$ and any principal submatrix of a positive semi-definite matrix is still positive semi-definite, it follows that

$$
\mathcal{H}_{1}^{\mathcal{K}} \cap \mathcal{H}_{2}^{\mathcal{K}} \subseteq \cup_{i \in \mathcal{K}}\left(\mathcal{H}_{1, i}^{\mathcal{K}} \cap \mathcal{H}_{2}^{\mathcal{K} \backslash\{i\}}\right)
$$

Since $\mathcal{H}_{1, i}^{\mathcal{K}} \subseteq \tilde{\mathcal{H}}_{1, i}^{\mathcal{K}}$ and the two events $\tilde{\mathcal{H}}_{1, i}^{\mathcal{K}}$ and $\mathcal{H}_{2}^{\mathcal{K} \backslash\{i\}}$ are independent, we have for any given $i \in \mathcal{K}$,

$$
P\left(\left\|x^{*}\right\|_{0}=k\right) \leq k C(n, k) P\left(\tilde{\mathcal{H}}_{1, i}^{\mathcal{K}}\right) P\left(\mathcal{H}_{2}^{\mathcal{K} \backslash\{i\}}\right),
$$

In the remaining of this section, we discuss how to bound the probabilities $P\left(\tilde{\mathcal{H}}_{1, i}^{\mathcal{K}}\right)$ and $P\left(\mathcal{H}_{2}^{\mathcal{K} \backslash\{i\}}\right)$ under different distribution assumptions.

\subsection{Concave Cumulative Distributions}

In this subsection, we focus on the scenario where the matrix $Q$ is generated as follows: first randomly generate its lower triangle and diagonal elements independently using the same cumulative distribution $G(\cdot)$; then symmetrize the matrix by assigning the upper triangle elements with their corresponding lower triangle elements. We assume that $G(\cdot)$ is continuous and concave in its support.

We first show how to bound the probability $P\left(\hat{\mathcal{H}}_{1, i}^{\mathcal{K}}\right)$, which interestingly can be derived from the probability bound established in Theorem 2.2.

Lemma 3.1. Let $Q_{\mathcal{K}} \in \Re^{k \times k}$ be an arbitrary principal sub-matrix of $Q \in$ $\Re^{n \times n}$, a symmetric matrix whose diagonal and lower diagonal elements are 
identically independently distributed with a continuous cumulative distribution function concave in its support. Then we have that

$$
P\left(\tilde{\mathcal{H}}_{1, i}^{\mathcal{K}}\right) \leq \frac{k(k-1)^{k-1}(n-k) !}{n !} .
$$

Proof. Recall (8) and set $U_{r}=Q_{i j_{r}}, r=1, \ldots, k-1$ for any $j_{r}$ with $\left\{j_{1}, \ldots, j_{r}\right\}=$ $\mathcal{K} \backslash\{i\}$, and $V_{r}=Q_{l_{r} l_{r}}, r=1, \ldots, n-k+1$ for any $l_{r}$ with $\left\{l_{1}, \ldots, l_{n-k+1}\right\}=$ $(\{1, \ldots, n\} \backslash \mathcal{K}) \cup\{i\}$. Let $\left[u_{r}\right]_{r=1}^{k-1}$ and $\left[v_{r}\right]_{r=1}^{n-k+1}$ be the corresponding order statistics in the ascending order. Conditioning on the position of $Q_{i i}$ in the order statistics of $V_{r}$, we have that for $k \geq 2$

$$
\begin{aligned}
P\left(\tilde{\mathcal{H}}_{1, i}^{\mathcal{K}}\right) & =P\left(\sum_{r=1}^{k-1} u_{r} \leq k v_{1}-Q_{i i}\right) \\
& =\sum_{j=1}^{n-k+1} P\left(\sum_{r=1}^{k-1} u_{r} \leq k v_{1}-v_{j}, Q_{i i}=v_{j}\right) \\
& =\sum_{j=1}^{n-k+1} P\left(Q_{i i}=v_{j}\right) P\left(\sum_{r=1}^{k-1} u_{r} \leq k v_{1}-v_{j}\right) \\
& \leq \frac{1}{n-k+1} k P\left(\sum_{r=1}^{k-1} u_{r} \leq k v_{1}\right) \\
& \leq \frac{1}{n-k+1} \frac{k(k-1)^{k-1}(n-k+1) !}{n !},
\end{aligned}
$$

where the last two inequalities follows from Theorem 2.2.

We next estimate the probability $P\left(\mathcal{H}_{2}^{\mathcal{K} \backslash\{i\}}\right)$. For this purpose, we present the following technical result.

Lemma 3.2. For a given symmetric matrix $Q$, if $Q-\lambda E$ is positive semidefinite for some $\lambda \in \Re$, then the projection matrix of $Q$ onto the null space of $e$ is positive semidefinite. Moreover, it holds

$$
Q_{i j} \leq \frac{1}{2}\left(Q_{i i}+Q_{j j}\right) \leq \max \left(Q_{i i}, Q_{j j}\right), \quad \forall i, j ;
$$

and

$$
Q_{i j} \leq \sqrt{Q_{i i} Q_{j j}}, \quad \forall i, j \in \mathcal{K}
$$

when $\lambda \geq 0$.

Proof. The first statement of the proposition follows directly from the fact that $Q-\lambda E$ is positive semidefinite, which further implies

$$
\hat{Q}=\left(\begin{array}{ll}
Q_{i i} & Q_{i j} \\
Q_{j i} & Q_{j j}
\end{array}\right)-\lambda\left(\begin{array}{cc}
1 & 1 \\
1 & 1
\end{array}\right) \succeq 0 .
$$

The positive semi-definiteness of the submatrix $\hat{Q}$ is equivalent to the following conditions: $Q_{i i}-\lambda \geq 0$ and

$$
\left(Q_{i j}-\lambda\right)^{2} \leq\left(Q_{i i}-\lambda\right)\left(Q_{j j}-\lambda\right),
$$

which implies that

$$
Q_{i j}-\lambda \leq \sqrt{\left(Q_{i i}-\lambda\right)\left(Q_{j j}-\lambda\right)} \leq \frac{\left(Q_{i i}-\lambda\right)+\left(Q_{j j}-\lambda\right)}{2} .
$$


Thus, the inequality (12) holds. If $\lambda \geq 0$, then $Q$ is the sum of two positive semi-definite matrices $(Q-\lambda E)$ and $\lambda E$ and thus is positive semi-definite as well, which further yields the inequality (13).

We now use the inequality (12) to provide a bound on the probability $P(\exists \lambda \in$ $\Re: Q-\lambda E \succeq 0)$, which can help to bound $P\left(\mathcal{H}_{2}^{\mathcal{K} \backslash\{i\}}\right)$.

Theorem 3.3. Let $Q \in \Re^{n \times n}$ be a symmetric matrix whose diagonal and lower diagonal elements are identically independently distributed with a continuous cumulative distribution function. Then it holds

$$
P(\exists \lambda \in \Re: Q-\lambda E \succeq 0) \leq \frac{2^{n}}{(n+1) !}
$$

Proof. From the last inequality in (12), conditioning on the event $\mathcal{A}=\left\{Q_{11} \geq\right.$ $\left.\ldots \geq Q_{n n}\right\}$, the event $\{\exists \lambda \in \Re: Q-\lambda E \succeq 0\}$ implies the following event

$$
\mathcal{B}=\left\{Q_{i j} \leq Q_{k k}, \quad \forall n \geq i \geq j \geq k \geq 1\right\} .
$$

Define a series of events

$$
\mathcal{B}_{k}=\left\{Q_{i k} \leq Q_{k k}, \quad \forall n \geq i>k\right\}
$$

We have that

$$
\mathcal{B}=\mathcal{A} \cap \cap_{k=1}^{n} \mathcal{B}_{k}
$$

Therefore,

$$
\begin{aligned}
P(\exists \lambda \in \Re: Q-\lambda E \succeq 0) & \leq P(\mathcal{B} \mid \mathcal{A}) \\
& =\frac{P(\mathcal{B})}{P(\mathcal{A})} \\
& =n ! \int_{-\infty}^{\infty} d G\left(u_{1}\right) \int_{-\infty}^{u_{1}} d G\left(u_{2}\right) \ldots \int_{-\infty}^{u_{n-1}} d G\left(u_{n}\right) \prod_{i=1}^{n} G\left(u_{i}\right)^{n-i} \\
& =n ! \int_{0}^{1} d t_{1} \int_{0}^{t_{1}} d t_{2} \ldots \int_{0}^{t_{n-1}} d t_{n} \prod_{i=1}^{n} t_{i}^{n-i} \\
& =n ! \prod_{i=1}^{n} \overline{1} \overline{i(i+1) / 2} \\
& =\frac{2^{n}}{(n+1) !},
\end{aligned}
$$

where the third equality follows by a variable transformation.

The above theorem can be improved for uniform/exponential distributions.

Theorem 3.4. If $Q \in \Re^{n \times n}$ be a symmetric matrix whose diagonal and lower diagonal elements are identically independently generated from the uniform distribution (i.i.u.d.) in $(0,1)$, then it holds

$$
P(Q \succeq 0) \leq \frac{2^{n}}{(n+1)^{n}} .
$$

If $Q \in \Re^{n \times n}$ be a symmetric matrix whose diagonal and lower diagonal elements are identically independently generated from the exponential distribution(i.i.e.d.) in $(0, \infty)$ with rate parameter 1 , then it holds

$$
P(Q \succeq 0) \leq \frac{2^{n}}{(n-1)^{n}} .
$$


Proof. If $Q$ is generated from i.i.u.d. in $(0,1)$, from (13) it follows

$$
\begin{aligned}
P(Q \succeq 0) & \leq P\left(Q_{i j} \leq Q_{i i}^{1 / 2} Q_{j j}^{1 / 2}, \forall 1 \leq i<j \leq n\right) \\
& =\int_{0}^{1} \cdots \int_{0}^{1} \prod_{i<j} t_{i}^{1 / 2} t_{j}^{1 / 2} d t_{1} \cdots d t_{n} \\
& =\int_{0}^{1} \cdots \int_{0}^{1} \prod_{i=1}^{n} t_{i}^{\frac{n-1}{2}} d t_{1} \cdots d t_{n}=\frac{2^{n}}{(n+1)^{n}} .
\end{aligned}
$$

In case of exponential distribution, from the first inequality in (12) we obtain

$$
\begin{aligned}
P(Q \succeq 0) & \leq P\left(Q_{i j} \leq \frac{Q_{i i}+Q_{j j}}{2}, \forall i<j\right) \\
& =\int_{0}^{\infty} \cdots \int_{0}^{\infty} \prod_{i<j}\left(1-\exp \left(-\frac{t_{i}+t_{j}}{2}\right)\right) \exp \left(-\sum_{i=1}^{n} t_{i}\right) d t_{1} \cdots d t_{n} \\
& =\int_{0}^{1} \cdots \int_{0}^{1} \prod_{i<j}\left(1-\sqrt{u_{i} u_{j}}\right) d u_{1} \cdots d u_{n} \\
& \leq \int_{0}^{1} \cdots \int_{0}^{1}\left(1-\frac{2 \sum_{i<j} \sqrt{u_{i} u_{j}}}{n^{2}-n}\right)^{\frac{n^{2}-n}{2}} d u_{1} \cdots d u_{n} \\
& \leq \int_{0}^{1} \cdots \int_{0}^{1}\left(1-\prod_{i=1}^{n} u_{i}^{\frac{1}{n}}\right)^{\frac{n^{2}-n}{2}} d u_{1} \cdots d u_{n} \\
& =n^{n} \int_{0}^{1} \cdots \int_{0}^{1}\left(1-\prod_{i=1}^{n} v_{i}\right)^{\frac{n^{2}-n}{2}} \prod_{i=1}^{n} v_{i}^{n-1} d u_{1} \cdots d u_{n} \\
& \leq n^{n} \int_{0}^{1} \cdots \int_{0}^{1}\left(1-\prod_{i=1}^{n} v_{i}\right)^{\frac{n^{2}-n}{2}} \prod_{i=1}^{n} v_{i}^{n-i} d u_{1} \cdots d u_{n} \\
& =\frac{n^{n}}{\frac{n^{2}-n}{2}+1} \int_{0}^{1} \cdots \int_{0}^{1}\left(1-\prod_{i=1}^{n-1} v_{i}\right)^{\frac{n^{2}-n}{2}+1} \prod_{i=1}^{n-1} v_{i}^{n-1-i} d u_{1} \cdots d u_{n-1} \\
& =\frac{n^{n}}{\prod_{i=1}^{n}\left(\frac{n^{2}-n}{2}+i\right)} \\
& \leq \frac{2^{n}}{(n-1)^{n}},
\end{aligned}
$$

where the second and third inequalities follow from the inequality of arithmetic and geometric means, the third equality from the variable transformation $u_{i}=$ $v_{i}^{n}(i=1, \ldots, n)$.

To build some intuition about the relative magnitudes of the probability bounds in Theorems 3.3 and 3.4, we use the well-known Stirling's approximation 
for large factorials, which states [35]

$$
\lim _{n \rightarrow \infty} \frac{n !}{\sqrt{2 \pi n}\left(\frac{n}{\exp (1)}\right)^{n}}=1 .
$$

It is clear that the bounds in Theorem 3.4 improve upon the one in Theorem 3.3 roughly by an order of $\exp (n)$.

Combining Theorem 3.3 and Lemma 3.1, we can obtain the following result.

Theorem 3.5. Assume that $Q \in \Re^{n \times n}$ is a symmetric matrix whose diagonal and lower diagonal elements are identically independently distributed with a continuous cumulative distribution function concave in its suppor. Let $x^{*}$ be one global optimal solution of problem (1). It holds for $k \geq 2$

$$
P\left(\left\|x^{*}\right\|_{0}=k\right) \leq \frac{(k-1)^{k-1} 2^{k-1}}{((k-1) !)^{2}} .
$$

Proof. From (10), Theorem 3.4 and Lemma 3.1, we obtain

$$
\begin{aligned}
P\left(\left\|x^{*}\right\|_{0}=k\right) & \leq k C(n, k) P\left(\tilde{\mathcal{H}}_{1, i}^{\mathcal{K}}\right) P\left(\mathcal{H}_{2}^{\mathcal{K} \backslash\{i\}}\right) \\
& \leq \frac{n !}{k !(n-k) !} \frac{k^{2}(k-1)^{k-1}(n-k) !}{n !} \frac{2^{k-1}}{k !} .
\end{aligned}
$$

Thus, the theorem holds.

We remark that comparing with the bound in [15] (presented in Theorem 2.4 ), for small $k$, the bound in the above theorem is not stronger. However, for reasonably large $k$, the new bound is tighter. Indeed, the Stirling's approximation implies that the new bound is roughly in the order of $\exp (-O(k \ln k))$ while the one in [15] is $\exp (-O(k))$.

When $Q=\left(M+M^{T}\right) / 2$ in which all elements of $M \in \Re^{n \times n}$ are identically independently distributed with a continuous cumulative distribution function concave in its support, it is much more complicated to provide an upper bound for $P(Q-\lambda E \succeq 0$ for some $\lambda \in \Re)$. However, Theorem 2.2 alone allows to provide an upper bound employing the approach in [15]. To compare with the result in Theorem 3.5, we present the bound in the follow theorem.

Theorem 3.6. Assume that $Q=\left(M+M^{T}\right) / 2$ in which all elements of $M \in$ $\Re^{n \times n}$ are identically independently distributed with a continuous cumulative distribution function concave in its support. We have that for $k \geq 2$

$$
P\left(\left\|x^{*}\right\|_{0}=k\right) \leq \frac{k(2 k-2)^{2 k-2}}{\prod_{j=1}^{k-2}(n+1+j)} .
$$

Proof. Define

$$
\mathcal{C}=\left\{\frac{1}{k-1} \sum_{j \in \mathcal{K} \backslash\{i\}} Q_{i j}<\min _{j \notin \mathcal{K} \backslash\{i\}} Q_{j j}\right\} .
$$

Note that $\tilde{\mathcal{H}}_{1}^{\mathcal{K}} \subseteq \mathcal{C}$. The term $\frac{1}{k-1} \sum_{j \in \mathcal{K} \backslash\{i\}} Q_{i j}$ can be treated as the average of $2(k-1)$ independent random variables and $\min _{j \notin \mathcal{K} \backslash\{i\}} Q_{j j}$ is the smallest order 
statistics of $n-k+1$ independent random variables, each of which has the same continuous cumulative distribution function concave in its support. Therefore,

$$
\begin{aligned}
P\left(\left\|x^{*}\right\|_{0}=k\right) & \leq k C(n, k) P\left(\tilde{\mathcal{H}}_{1, i}^{\mathcal{K}}\right) \\
& \leq k C(n, k) P(\mathcal{C}) \\
& \leq k(2 k-2)^{2 k-2} \frac{n !(n-k+1) !}{k !(n-k) !(n+k-1) !} \\
& \leq \frac{k(2 k-2)^{2 k-2}}{\prod_{j=1}^{k-2}(n+1+j)}
\end{aligned}
$$

which gives the bound in the theorem.

We remark that for fixed $k$, the above result essentially excludes the possibility of having global optimal solutions with more than two positive elements when $n$ is large.

\subsection{Normal Distribution}

In this subsection, we discuss how to estimate the probability $P\left(\left\|x^{*}\right\|_{0}=k\right)$ when $Q$ is generated from an identically independent normal distribution $\mathcal{N}(0,1)$ (i.i.n.d.). We focus on the case in which $Q$ is generated as follows: first generate all elements of a matrix $M \in \Re^{n \times n}$ identically independently from the standard normal distribution $\mathcal{N}(0,1)$; then set $Q=\left(M+M^{T}\right) / 2$. In such a case, the diagonal elements and the off-diagonal elements of $Q$ have different variances. The matrix $Q$ is referred to as the Gaussian Orthogonal Ensemble (GOE) [31] in the literature of random matrix theory.

Again our task is to bound the probability $P\left(\tilde{\mathcal{H}}_{1, i}^{\mathcal{K}}\right)$ and $P\left(\mathcal{H}_{2}^{\mathcal{K} \backslash\{i\}}\right)$. Unfortunately, the analysis in the previous subsection does not carry over to the case with Normal distribution. In fact, though Theorem 3.3 works for general distributions, Lemma 3.1 is only established for distributions with concave cumulative distribution functions.

To bound the probability $P\left(\tilde{\mathcal{H}}_{1, i}^{\mathcal{K}}\right)$, we need the following key technical result. Its proof is quite involved and presented in the appendix.

Theorem 3.7. Let $U_{i} \sim N(0,1)(i=1, \ldots, k)$ be independent and $V_{1}$ be the smallest order statistics of $n$ independent standard normal random variables independent of $U_{i}$. There exists a constant $\eta>0$ such that for any $1 \leq k \leq n$,

$$
\operatorname{Pr}\left(\frac{1}{k} \sum_{i=1}^{k} U_{i} \leq V_{1}\right) \leq \eta^{k}\left(\ln \left(\frac{n+k}{k}\right)\right)^{\left\lceil\frac{k-1}{2}\right\rceil} B(k, n+1) .
$$

The above theorem allows us to bound the probability $P\left(\tilde{\mathcal{H}}_{1, i}^{\mathcal{K}}\right)$.

Theorem 3.8. If $Q \in \Re^{n \times n}$ is GOE, we have that for $k \geq 2$.

$$
P\left(\tilde{\mathcal{H}}_{1, i}^{\mathcal{K}}\right) \leq\left(\eta^{2} \ln \left(\frac{n+k-1}{2 k-2}\right)\right)^{k-1} B(2 k-2, n-k+2) .
$$


Proof. Since $Q$ is GOE, we have that $G=\left(M+M^{T}\right) / 2$ with each component of $M \in \Re^{n \times n}$ being i.i.n.d. Similar to the proof of Theorem 3.6, we have that

$$
\tilde{\mathcal{H}}_{1, i}^{\mathcal{K}} \subseteq \mathcal{C}=\left\{\frac{1}{k-1} \sum_{j \in \mathcal{K} \backslash\{i\}} Q_{i j}<\min _{j \notin \mathcal{K} \backslash\{i\}} Q_{j j}\right\} .
$$

The term $\frac{1}{k-1} \sum_{j \in \mathcal{K} \backslash\{i\}} Q_{i j}$ can be treated as the average of $2(k-1)$ independent standard normal random variables and $\min _{j \notin \mathcal{K} \backslash\{i\}} Q_{j j}$ is the smallest order statistics of $n-k+1$ independent standard normal random variables. Therefore, the bound on $P(\mathcal{C})$ and thus $P\left(\tilde{\mathcal{H}}_{1, i}^{\mathcal{K}}\right)$ follow by using Theorem 3.7.

The above theorem can be combined with the bound of $P\left(\mathcal{H}_{2}^{\mathcal{K} \backslash\{i\}}\right)$ in Theorem 3.3 to provide an upper bound of the probability $P\left(\left\|x^{*}\right\|_{0}=k\right)$. Interestingly, a much stronger bound of $P\left(\mathcal{H}_{2}^{\mathcal{K} \backslash\{i\}}\right)$ is available from the random matrix theory. To present the bound, note that the GOE enjoys the following nice properties [31]:

P.1 The ensemble is invariant under the transformation

$$
Q \rightarrow W Q W^{T}
$$

where $W$ is any real orthogonal matrix;

P.2 The elements $Q_{i j}, i \leq j$, are statistically independent.

P.3 It was shown in [18] that for the GOE,

$$
P(Q \succeq 0) \leq \exp ^{-\frac{n^{2}}{4}} .
$$

To apply the above properties of GOE, choose a specific orthogonal matrix $W$ such that its last row $W_{n,:}=e^{T} / \sqrt{n}$. It follows immediately that the principal submatrix of $W Q W^{T}$ with the first $n-1$ rows and columns, $W_{1: n-1,:} Q W_{1: n-1,:}^{T}$, is GOE and the property P.3 implies that

$$
P\left(W_{1: n-1,:} Q W_{1: n-1,:}^{T} \succeq 0\right) \leq \exp \left(-\frac{(n-1)^{2}}{4}\right) .
$$

Since

$$
W_{1: n-1,:}(Q-\lambda E) W_{1: n-1,:}^{T}=W_{1: n-1,:} Q W_{1: n-1,:}^{T}, \forall \lambda \in \Re,
$$

we immediately obtain the following result.

Theorem 3.9. If $Q \in \Re^{n \times n}$ is the GOE generated from (i.i.n.d.), then it holds

$$
P(\exists \lambda \in \Re: Q-\lambda E \succeq 0) \leq \exp \left(-\frac{(n-1)^{2}}{4}\right) .
$$

Combing Theorems 3.8 and 3.9, we have the following result. 
Theorem 3.10. Assume that $Q \in \Re^{n \times n}$ is GOE. Let $x^{*}$ be one global optimal solution of problem (1). We have that for $k \geq 2$,

$P\left(\left\|x^{*}\right\|_{0}=k\right) \leq \frac{(2 k-3) !}{(k-1) !(n+1)^{k-2}}\left(\eta^{2} \ln \left(\frac{n+k-1}{2 k-2}\right)\right)^{k-1} \exp \left(-\frac{(k-2)^{2}}{4}\right)$

Proof. The result follows from (10), Theorem 3.8, Lemma 3.9 and the fact that $B(n, k)=\frac{(n-1) !(k-1) !}{(n+k-1) !}$ and

$$
\frac{n !(n-k+1)}{(n+k-1) !} \leq \frac{1}{(n+1)^{k-2}} .
$$

The above theorem implies that one can essentially exclude the possibility of global optimal solutions with three or more positive elements for large $n$.

\section{Sparse Approximate Solutions to Convex StQPs and Extensions}

In this section we investigate the existence of sparse approximate solutions to StQPs with a symmetric positive semi-definite matrix $Q \in \Re^{n \times n}$. Different from the previous section, we focus on deterministic $Q$. Without loss of generality, we can further assume that all the matrix elements are positive. In fact, the optimal solution set of problem (1) is the same when $Q$ is changed by adding $\lambda E$ for any $\lambda$. Under such an assumption, we can decompose the matrix into the following form

$$
Q=V^{T} V, \quad V=\left[v_{1} ; v_{2}, \cdots, v_{n}\right],
$$

where the $i$-th column of $V$ represent a data point in $\Re^{d}$ for some $d$ with $\operatorname{rank}(Q) \leq d \leq n$, and $Q_{i j}=v_{i}^{T} v_{j}>0$ for $i, j=1, \ldots, n$. It follows immediately

$$
x^{T} Q x=\|V x\|_{2}^{2},
$$

and the StQP reduces to a problem of finding the shortest distance between the origin in $\Re^{d}$ and the polytope $P(V)$ defined by

$$
P(V)=\left\{v \in \Re^{d}: v=V x,\|x\|_{1}=1, x \geq 0 .\right\}
$$

In other words, the StQP amounts to solving the following optimization problem

$$
\min _{v \in P(V)}\|v\|
$$

Since $Q_{i j}=v_{i}^{T} v_{j}>0$ for $i, j=1, \ldots, n$, it must hold $0 \notin P(V)$. Therefore, the optimal solution to problem (18) must be achieved at a facet of the polytope $P(V)$, which implies that the optimal solution $v$ can be represented as a convex combination of at most $d$ points. If $d<<n$, this indicates that the corresponding StQP has a very sparse optimal solution. 
We next consider the case where $d$ is very large and in the same order of $n$. We first cite a well-known result for embedding by Johnson and Lindenstrauss [25, 27].

Lemma 4.1. Given $\epsilon>0$ and an integer $n$. Let $k$ be a positive integer such that $k \geq k_{0}=\mathcal{O}\left(\epsilon^{-2} \log (n)\right)$. For every set $P$ of $n$ points in $\Re^{d}$, there exists a mapping $h: \Re^{d} \rightarrow \Re^{k}$ such that

$$
(1-\epsilon)\|u-v\|^{2} \leq\|h(u)-h(v)\|^{2} \leq(1+\epsilon)\|u-v\|^{2}, \quad \forall u, v \in P .
$$

In particular, in [2], Achlioptas proved the following interesting result.

Theorem 4.2. Let $P$ be an arbitrary set of $n$ points in $\Re^{d}$ represented by a matrix $A \in \Re^{d \times n}$. Given $\epsilon, \beta>0$ and let

$$
k_{0}=\frac{4+2 \beta}{\epsilon^{2} / 2-\epsilon^{3} / 3} \log (n) .
$$

For integer $k>k_{0}$, let $R \in \Re^{k \times d}$ be a random matrix with $R(i, j)=r_{i j}$, where $r_{i j}$ are independent random variables from either one of the following two probability distributions

$$
\begin{array}{r}
r_{i j}=\left\{\begin{array}{ccc}
1 & \text { with probability } & \frac{1}{2} \\
-1 & \ldots & \frac{1}{2}
\end{array}\right. \\
r_{i j}=\sqrt{3} \times\left\{\begin{array}{ccc}
1 & \text { with probability } & \frac{1}{6} \\
0 & \ldots & \frac{2}{3} \\
-1 & \ldots & \frac{1}{6}
\end{array}\right.
\end{array}
$$

Let

$$
M=\frac{1}{\sqrt{k}} R A .
$$

Let $h: \Re^{d} \rightarrow \Re^{k}$ map the $i$-th column of $A$ to the $i$-th column of $M$. With probability at least $1-n^{-\beta}$, we have

$$
(1-\epsilon)\|u-v\|^{2} \leq\|h(u)-h(v)\|^{2} \leq(1+\epsilon)\|u-v\|^{2}, \quad \forall u, v \in P .
$$

To apply the above theorem to the StQP problem where $Q=V^{T} V$, we can first augment the matrix $V$ by adding one more column (the origin in $\Re^{d}$ ). We then use the above-described random projection method to the augmented data set $V$ into a low dimensional space of size $\mathcal{O}\left(\frac{\log n}{\epsilon^{2}}\right)$. Next we solve problem (18) with the projected data set excluding the origin. By using Theorem 4.2, one can show that with a high probability, the solution from the projected StQP in a low dimensional space provides an $(1+\epsilon)$-approximation to the StQP in the original space, which indicates with probability at least $1-n^{-\beta}$, there exists an $(1+\epsilon)$-approximate solution to the original StQP whose sparsity is $\mathcal{O}\left(\frac{\log n}{\epsilon^{2}}\right)$.

We close this section by discussing potential extensions of the results in the previous sections to other classes of quadratic optimization problems. To start, let us consider a generic QP model defined by

$$
\begin{aligned}
\min & x^{T} Q x+r^{T} x \\
\text { s.t. } & A x=b, \quad x \geq 0 .
\end{aligned}
$$


Here $A \in \Re^{m \times n}, b \in \Re^{m}$. Let us consider the case that the feasible set, denoted by $\mathcal{F}=\{x: A x=b, x \geq 0\}$, is a bounded polytope whose vertices belongs to the following set defined by

$$
\mathcal{S}=\left\{s_{i}: i=1, \ldots, S .\right\}
$$

From the above definition, for every $x \in \mathcal{F}$, there exists $\alpha_{i} \geq 0, i=1, \cdots, S$ such that

$$
x=\sum_{i=1}^{S} \alpha_{i} s_{i}, \quad \sum_{i=1}^{S} \alpha_{i}=1 .
$$

Therefore, we can rewrite problem (21) as another StQP as follows

$$
\begin{array}{ll}
\min & \alpha^{T} \hat{Q} \alpha+\hat{r}^{T} \alpha=\alpha^{T} \bar{Q} \alpha \\
\text { s.t. } & \sum_{i=1}^{S} \alpha_{i}=1, \quad \alpha \geq 0,
\end{array}
$$

where

$$
\hat{Q}_{i j}=s_{i}^{T} Q s_{j}, \quad \hat{r}_{i}=r^{T} s_{i}, \quad \bar{Q}=\hat{Q}+\left(\hat{r} e_{S}^{T}+e_{S} \hat{r}^{T}\right) / 2 .
$$

If we assume that the matrix $\bar{Q}$ is generated from a certain distribution, then by using the results in the previous sections, we can conclude that the global optimal solution of problem (22) is very sparse. Suppose that the global optimal solution to problem (22) has $k$ positive elements, then the globally optimal solution to problem (21) can be written as a convex combination of $k$ vertices of $\mathcal{F}$. Since $A \in \Re^{m \times n}$, every vertex of $\mathcal{F}$ has at most $m$ positive elements. It follows immediately that the global optimal solution to problem (21) has at most $k m$ positive elements. Therefore, when $m$ is a very small integer and the elements of the matrix $\bar{Q}$ are generated from a certain distribution, we can conclude that with a high probability, the global optimal solution to problem (21) is very sparse.

A particular example that worths mentioning is the mean-variance model introduced in [30] where

$$
\mathcal{F}=\left\{x: e^{T} x=1, \mu^{T} x=\bar{\mu}, x \geq 0\right\} .
$$

It has been observed for a long time that the optimal solution of the meanvariance model is dominated by only a few assets [16], which corresponds to a very sparse exact or approximate solution for the underlying QP. Note that for the mean-variance model, we have $m=2$. Therefore, the results in this paper provides a potential interesting interpretation for the observed phenomena with respect to the mean-variance model.

\section{Conclusions}

In this paper, we consider the so-called standard quadratic programming problems that arise from various applications. Under certain probability models 
of the input data matrix, we show by performing probability analysis based on the first-order and second-order optimality conditions of the underling QP that there is a very high probability that the optimal solution to a random StQP has only a few nonzero elements. Our results significantly improve the corresponding ones in [15] employing the first-order optimality condition when the elements of the input data matrix are generated by continuous distribution functions concave in their supports. More importantly, we show that when the input data matrix is GOE, the global optimal solutions of StQP (1) essentially have at most two nonzero positive elements for large $n$. To prove the result, we establish a key technical result which provides an upper bound of the probability of the event that the average of $k$ independent standard normal random variables is no more than the smallest order statistics of $n$ independent standard normal random variables, which we believe can be useful in other settings. We also show that if the input data matrix is a deterministic positive semi-definite matrix, with high probability a sparse approximate solution can be found.

Our results raise up several interesting research questions. First, we would like to know how tight our bounds are. Second, can we establish general and/or better results regarding the positive semi-definiteness of a randomly generated symmetric matrix? More specifically, can we improve the bounds in Theorems 3.3, 3.4? Can we provide similar bounds like we do for GOE when $Q=\left(M+M^{T}\right) / 2$ with each element of $M$ being identically independently distributed with a general distribution? Third, our analysis for normal distribution only works for GOE. Can we provide similar bounds if $Q$ is symmetric with identically independent distributed diagonal and lower triangle elements? Finally, we point out that though the results in this work can provide a partial interpretation regarding the mean-variance model for portfolio selection, it is far from satisfactory since we use some assumptions on the data matrix $\bar{Q}$, which is constructed from the original input data matrix $Q$ and the vertices of the constraint set $\mathcal{F}$. It will be of interest to explore whether we can establish similar results based on characteristics of the input data matrices $Q$ and the coefficient data matrix $A$ directly.

\section{Appendix: Proof of Theorem 3.7}

The proof is done by three steps. In the first step, we write down the probability as an integral formula and divide the integral into two parts. One part of the integral can be easily upper bounded by a function exponentially decaying in $n$. In the second step, we provide an upper bound for another part of the integral using a tight approximation of the complementary cumulative distribution function of the standard normal distribution. In the third step, we show that the upper bounds on the two parts of the integral have consistent orders.

Step 1

Denote $f(u)=\exp \left(-u^{2} / 2\right)$. Let $\phi(u)=\frac{1}{\sqrt{2 \pi}} f(u), \Phi(u)=\int_{-\infty}^{c} \phi(\xi) d \xi$ and $\Phi^{c}(u)=1-\Phi(u)$ be the pdf, cdf and complementary cdf of the standard normal 
distribution. It is clear that

$$
\operatorname{Pr}\left(\frac{1}{k} \sum_{i=1}^{k} U_{i} \leq V_{1}\right)=\frac{\sqrt{k}}{\sqrt{2 \pi}} I_{n, k}(-\infty, \infty),
$$

where

$$
I_{n, k}(l, u)=\int_{l}^{u} f(\sqrt{k} u) \Phi^{c}(u)^{n} d u .
$$

To estimate $I_{0}(-\infty, \infty)$, we divide the integral into two parts.

$$
I_{n, k}(-\infty, \infty)=I_{n, k}(-\infty,-1)+I_{n, k}(-1, \infty) .
$$

Since $\Phi^{c}(u)$ is decreasing in $u$,

$$
I_{n, k}(-1, \infty) \leq \Phi^{c}(-1)^{n}=\Phi(1)^{n} .
$$

\section{Step 2}

It suffices to estimate $I_{n, k}(-\infty,-1)$. For this purpose, we use the following approximation of $\Phi^{c}(u)$ for $u \geq 0$ (see 7.1 .13 in [1]).

Lemma 5.1. For $u \geq 0$,

$$
\frac{2}{u+\sqrt{u^{2}+4}} \phi(u)<\Phi^{c}(u) \leq \frac{2}{u+\sqrt{u^{2}+8 / \pi}} \phi(u) .
$$

Using the above lemma, we can immediately show that for $u \geq 1$,

$$
\frac{1}{\rho u} f(u)<\Phi^{c}(u) \leq \frac{1}{\rho^{\prime} u} f(u)
$$

where $\rho^{\prime}=\sqrt{2 \pi}$ and $\rho=\frac{1+\sqrt{5}}{2} \sqrt{2 \pi}$.

We now derive an upper bound of $I_{n, k}(-\infty,-1)$ using Lemma 5.1.

$$
\begin{aligned}
I_{n, k}(-\infty,-1) & =\frac{\sqrt{k}}{\sqrt{2 \pi}} \int_{-\infty}^{-1} f(\sqrt{k} u) \Phi^{c}(u)^{n} d u \\
& =\frac{\sqrt{k}}{\sqrt{2 \pi}} \int_{1}^{\infty} f(\sqrt{k} u)\left(1-\Phi^{c}(u)\right)^{n} d u \\
& \leq \int_{1}^{\infty} f(\sqrt{k} u)\left(1-\frac{1}{\rho u} f(u)\right)^{n} d u \\
& =\int_{v_{0}}^{\infty} f(\sqrt{k} v)(1-f(v))^{n}(\rho u(v))^{k} \frac{v}{u(v)+1 / u(v)} d v
\end{aligned}
$$

where the second equality follows from the symmetry of the Normal distribution, the first inequality from Lemma 5.1, the last equality is derived by a variable change with $v=\sqrt{2\left(u^{2} / 2+\ln (\rho u)\right)}$ and $v_{0}=\sqrt{1+2 \ln (\rho)}$. Since $u \leq v$, we have from the above formula that

$$
I_{n, k}(-\infty,-1) \leq \rho^{k} J_{n, k}
$$

where

$$
J_{n, k}=\int_{v_{0}}^{\infty} f(\sqrt{k} v)(1-f(v))^{n} v^{k} d v
$$


Making a variable change with $z=f(v)$, we have that

$$
J_{n, k}=2^{\frac{k-1}{2}} \int_{0}^{z_{0}} z^{k-1}(1-z)^{n}(-\ln z)^{\frac{k-1}{2}} d z,
$$

where $z_{0}=f\left(v_{0}\right)<1$.

To provide an upper bound for $J_{n, k}$, we analyze the derivatives of the beta function. Define for a natural number $i$,

$$
\tau_{i}=(-1)^{i} \frac{d^{i} B(k, n+1)}{d k^{i}} .
$$

Note that

$$
\tau_{i}=\int_{0}^{1} z^{k-1}(1-z)^{n}(-\ln z)^{i} d z .
$$

Since $B(k, n+1)=\frac{\Gamma(k) \Gamma(n+1)}{\Gamma(k+n+1)}$ with the Gamma function

$$
\Gamma(z)=\int_{0}^{\infty} t^{z-1} e^{-t} d t
$$

we have that

$$
\tau_{1}=-B(k, n+1)\left(\psi_{0}(k)-\psi_{0}(k+n+1)\right)
$$

where

$$
\psi_{i}(z)=\frac{d^{i+1} \ln \Gamma(z)}{d z^{i+1}}=(-1)^{i+1} i ! \sum_{l=0}^{\infty} \frac{1}{(z+l)^{i+1}}
$$

is the polygamma function (for the definitions and relationships of the special functions, see Chapter 6 in [1]). Therefore,

$$
\tau_{i}=\sum_{j=0}^{i-1} C(i-1, j)(-1)^{i-j}\left(\psi_{i-1-j}(k)-\psi_{i-1-j}(k+n+1)\right) \tau_{j}
$$

We now provide an upper bound on $\tau_{i}$. From the above recursion, we have that for any $i-j>1$ and $i \leq k / 2$,

$$
\begin{aligned}
C_{i-1}^{j}(-1)^{i-j}\left(\psi_{i-1-j}(k)-\psi_{i-1-j}(k+n+1)\right) & =\frac{(i-1) !}{j !} \sum_{l=0}^{n} \frac{1}{(k+l)^{i-j}} \\
& \leq \frac{(i-1) !}{j !} \frac{1}{k^{i-j-2}} \sum_{l=0}^{\infty} \overline{(k+l)(k+l-1)} \\
& =\frac{(i-1) !}{j !} \frac{1}{k^{i-j-2}} \frac{1}{k-1} \\
& \leq\left(\frac{1}{2}\right)^{i-j-1} .
\end{aligned}
$$

Thus, we have

$$
\begin{aligned}
\tau_{i} & \leq \tau_{i-1} \sum_{l=0}^{n} \frac{1}{k+l}+\sum_{j=0}^{i-2} \tau_{j}\left(\frac{1}{2}\right)^{i-j-1} \\
& \leq \max _{j=0, \ldots, i-1} \tau_{j}\left(1+\sum_{l=0}^{n} \frac{1}{k+l}\right) \\
& \leq \max _{j=0, \ldots, i-1} \tau_{j}\left(\ln (n+k)-\ln (k)+\frac{1}{k}+1\right) \\
& \leq\left(\ln \left(\frac{\exp (2)(n+k)}{k}\right)\right)^{i} \tau_{0} \\
& =\left(\ln \left(\frac{\exp (2)(n+k)}{k}\right)\right)^{i} B(k, n+1) .
\end{aligned}
$$


We now use the above result on $\tau_{i}$ to bound $J_{n, k}$ :

$$
\begin{aligned}
J_{n, k} & =2^{\frac{k-1}{2}} \int_{0}^{z_{0}} z^{k-1}(1-z)^{n}(-\ln z)^{\frac{k-1}{2}} d z \\
& \leq 2^{\frac{k-1}{2}} \int_{0}^{z_{0}} z^{k-1}(1-z)^{n}\left[(-\ln z)^{\left\lceil\frac{k-1}{2}\right\rceil}+(-\ln z)^{\left\lfloor\frac{k-1}{2}\right\rfloor}\right] / 2 d z \\
& \leq 2^{\frac{k-1}{2}} \int_{0}^{1} z^{k-1}(1-z)^{n}\left[(-\ln z)^{\left\lceil\frac{k-1}{2}\right\rceil}+(-\ln z)^{\left\lfloor\frac{k-1}{2}\right\rfloor}\right] / 2 d z \\
& \leq \frac{\sqrt{k}}{\sqrt{2 \pi}} 2^{\frac{k-1}{2}}\left[\left(\ln \left(\frac{\exp (2)(n+k)}{k}\right)\right)^{\left\lceil\frac{k-1}{2}\right\rceil}+\left(\ln \left(\frac{\exp (2)(n+k)}{k}\right)\right)^{\left\lfloor\frac{k-1}{2}\right\rfloor}\right] / 2 B(k, n+1) \\
& \leq \frac{\sqrt{k}}{\sqrt{2 \pi}} 2^{\frac{k-1}{2}}\left(\ln \left(\frac{\exp (2)(n+k)}{k}\right)\right)^{\left\lceil\frac{k-1}{2}\right\rceil} B(k, n+1),
\end{aligned}
$$

where the first inequality follows from the convexity of $x^{n}$ in $n$ and the last inequality holds since the $\ln$ term is greater than 1 .

\section{Step 3}

The above analysis implies that

$$
\operatorname{Pr}\left(\frac{1}{k} \sum_{i=1}^{k} U_{i} \leq V_{1}\right) \leq \rho^{k} J_{n, k}+\Phi(1)^{n} .
$$

When $k$ is fixed as a constant, the first term in the above inequality dominates the second term. However, when $k$ is large, their relationship is less clear. We now show that there exists a constant $\gamma \geq 1$ such that $\Phi(1)^{n}$ is dominated by $\gamma^{k} B(k, n+1)$.

For this purpose, we use the Stirling's formula $n !=\sqrt{2 \pi n}\left(\frac{n}{e}\right)^{n} e^{\lambda_{n}}$ with $\frac{1}{12 n+1}<\lambda_{n}<\frac{1}{12 n}$ (see 6.1.37 in [1]). We have that for $k>1$,

$$
\begin{aligned}
\ln B(k, n+1)= & \ln \frac{(k-1) ! n !}{(n+k) !} \\
\geq & \ln \sqrt{\frac{2 \pi(k-1) n}{(n+k)}}+(k-1) \ln (k-1)+n \ln n-(n+k) \ln (n+k) \\
= & \ln \sqrt{\frac{2 \pi n}{(n+k)(k-1)}}+k \ln (1-1 / k)+k[-\ln (1+n / k)-n / k \ln (1+k / n)] \\
\geq & \ln \sqrt{\frac{2 \pi}{2 k}}+k \ln (1-1 / k)+n \ln \Phi(1) \\
& -k \max _{0 \leq \alpha \leq 1}\{\ln (1+1 / \alpha)+\ln [(1+\alpha) \Phi(1)] / \alpha\} \\
= & \ln \sqrt{\frac{2 \pi}{2 k}}+k \ln (1-1 / k)-k \ln \delta+n \ln \Phi(1),
\end{aligned}
$$

where the first inequality follows from the Stirling's formula, and

$$
\delta=\max _{\alpha \in[0,1]}(1+1 / \alpha)[(1+\alpha) \Phi(1)]^{1 / \alpha} .
$$

Therefore, for $k>1$,

$$
\Phi(1)^{n} \leq \sqrt{\frac{2 k}{2 \pi}}(1-1 / k)^{-k} \delta^{k} B(k, n+1),
$$

and hence

$$
\operatorname{Pr}\left(\frac{1}{k} \sum_{i=1}^{k} U_{i} \leq V_{1}\right) \leq \frac{\sqrt{k}}{\sqrt{2 \pi}}\left(2^{\frac{k-1}{2}} \rho^{k}\left(\ln \left(\frac{\exp (2)(n+k)}{k}\right)\right)^{\left\lceil\frac{k-1}{2}\right\rceil}+\sqrt{2}(1-1 / k)^{-k} \delta^{k}\right) B(k, n+1)
$$


Using Mathematica, we observe that $\delta \leq 6.4$. It is clear that there exists a constant $\eta>0$ such that for any $k>1$,

$$
P\left(\frac{1}{k} \sum_{i=1}^{k} U_{i} \leq V_{1}\right) \leq \eta^{k}\left(\ln \left(\frac{\exp (2)(n+k)}{k}\right)\right)^{\left\lceil\frac{k-1}{2}\right\rceil} B(k, n+1) .
$$

Apparently, the $\exp (2)$ term inside ln can be combined with the $\eta$ term. For $k=1$, we have that

$$
P\left(\frac{1}{k} \sum_{i=1}^{k} U_{i} \leq V_{1}\right)=\frac{1}{n+1}=B(k, n+1),
$$

and thus Theorem 3.7 holds.

\section{References}

[1] Abramowitz, M. and Stegun, I.A. 1972. Handbook of mathematical functions: with formulas, graphs, and mathematical tables. Drover, New York.

[2] D. Achlioptas. Database-friendly random projections. In: Proc. 20-th Annual ACM SIGACT-SIGMOD-SIGART Symposium on Principles of Database Systems, pp. 274281, 2001.

[3] I. Barany, S. Vempala and A. Vetta. Nash equilibria in random games. Random Structures and Algorithms, 31(4), pp 391-405, 2007.

[4] R. Beier and B. Vöcking. Random knapsack in expected polynomial time. Proceedings of the thirty-fifth annual ACM symposium on Theory of computing, 232-241, 2003.

[5] A. Berman and R.J. Plemmons. Nonnegative Matrices in the Mathematical Sciences. SIAM Classics in Applied Math., Philadelphia, 1994.

[6] I.M. Bomze, On standard quadratic optimization problems, J. Global Optimization, 13(1998), pp. 369-387.

[7] I.M. Bomze, M. Dür, E. de Klerk, C. Roos, A.J. Quist and T. Terlaky, On copositive programming and standard quadratic optimization problems, J. Global Optim. 18 (2000), pp. 301-320.

[8] I.M. Bomze and E. de Klerk, Solving standard quadratic optimization problems via linear, semidefinite and copositive programming, J. Global Optim. 24 (2002), pp. 163-185.

[9] I.M. Bomze, M. Locatelli and F. Tardella, New and old bounds for standard quadratic optimization: dominance, equivalence and incomparability, Math. Programming, 1(115), pp. 31-64, 2008. 
[10] A. M. Bruckstein, M. Elad and M. Zibulevsky, A non-negative and sparse enough solution of an underdetermined linear system of equations is unique. Preprint, 2007. See ( http://www-dsp.rice.edu/cs).

[11] S. Burer, K.M. Anstreicher and M. Dür. The Difference Between 5x5 Doubly Nonnegative and Completely Positive Matrices. Linear Algebra and Its Applications, vol 431, 1539-1552, 2009.

[12] E. Candès and M. Wakin, An introduction to compressive sampling. IEEE Signal Processing Magazine, 25(2), pp. 21 - 30, 2008.

[13] E. Candès, J. Romberg and T. Tao, Robust uncertainty principles: Exact signal reconstruction from highly incomplete frequency information. IEEE Trans. on Information Theory, 52(2) pp. 489 - 509, 2006.

[14] E. Candés, X.D. Li, Y. Ma and J. Wright. Robust Principal Component Analysis? Preprint, December 2009. http://perception.csl.uiuc.edu/matrixrank/references.html.

[15] X. Chen, J. Peng and S. Zhang. Sparse Solutions to Random Standard Quadratic Optimization Problems. To appear in Mathematical Programming, 2012.

[16] G. Cornuejols and R. Tütüncü. Optimization Methods in Fiance. Cambridge University Press, 2007.

[17] H.A. David and H. N. Nagaraja. Order Statistics. John Wiley \& Sons, NJ, 2003.

[18] D.S. Dean and S.N. Majumdar. Extreme value statistics of eigenvalues of Guassian random matrices. Physical Review E 77, 041108.1-041108.12, 2008 .

[19] D. Donoho, Compressed sensing. IEEE Trans. on Information Theory, 52(4), pp. 1289 - 1306, 2006.

[20] L.E. Gibbons, D.W. Hearn, P. Pardalos and M. V. Ramana. Continuopus characterizations of the maximal clique problem. Mathematics of Operations Research, 22 (1997), 754-768.

[21] A. V. Goldberg and A. Marchetti-Spaccamela. On finding the exact solution of a zero-one knapsack problem, Proceedings of the sixteenth annual ACM symposium on Theory of computing, 359-368, December 1984.

[22] M. Grant, S. Boyd and Y. Ye. CVX Users' Guide, 2007. http://www.stanford.edu/ ${ }^{\sim}$ boyd/cvx/cvx_usrguide.pdf

[23] W. W. Hager, P. M. Pardalos, I. M. Roussos and H. D. Sahinoglou. Active constraints, indefinite quadratic test problems, and complexity. J. Opt. Theo. \& Appl. , 68, 499-511, 1991. 
[24] J. Hu, J.E. Mitchell and J.S. Pang. An LPCC Approach to Nonconvex Quadratic Programs. Technical report, Department of Mathematical Sciences, Rensselaer Polytechnic Institute, Troy, New York 12180-3590, 2008.

[25] P. Indyk and R. Motwani. Approximate nearest neighbors: Towards removing the curse of dimensionality. In Proc. 30th Annu. ACM Sympos. Theory Comput., 604613, 1998.

[26] T. Irabaki and N. Katoh, Resource Allocation Problems: Algorithmic Approaches. MIT Press, Cambridge, 1988.

[27] W. B. Johnson and J. Lindenstrauss. Extensions of lipschitz mapping into hilbert space. Contemporary Mathematics, 26:189206, 1984.

[28] E. de Klerk and D.V. Pasechnik, Approximation of the stability number of a graph via copositive programming, SIAM J. Optim. 12 (2002), pp. 875-892.

[29] W. Kaplan, A test for copositive matrices, Linear Algebra Appl. 313 (2000), pp 203-206.

[30] H.M. Markowitz, Portfolio selection, J. Finance, 7(1952), pp. 77-91.

[31] M.L. Mehta. Random Matrices. London, Academic Press, 1991.

[32] L. Mukherjee, V. Singh, J. Peng and C. Hinrichs. Learning Kernels for variants of Normalized Cuts: Convex Relaxations and Applications, Proceedings of Conference on Computer Vision and Pattern Recognition (CVPR), San Francisco, June 13-18, 2010.

[33] K.G. Murty and S.N. Kabadi, Some NP-complete problems in quadratic and nonlinear programming, Math. Program. 39 (1987), pp. 117-129.

[34] B. Recht, M. Fazel and P.A. Parrilo. Guaranteed Minimum-Rank Solutions of Linear Matrix Equations via Nuclear Norm Minimization. http://arxiv.org/abs/0706.4138.

[35] D. Romik. Stirlings Approximation for $n$ !: The Ultimate Short Proof? The American Mathematical Monthly, Vol. 107(6), 556557, 2000.

[36] A. Scozzari and F. Tardella. A clique algorithm for standard quadratic programming, Discrete Applied Math., 156(2008), 2439-2448.

[37] K.C. Toh, M.J. Todd, and R.H. Tütüncü, SDPT3 - a Matlab software package for semidefinite programming. Optimization Methods and Software, 11 (1999), pp. 545-581.

[38] H. Väliaho, Quadratic programming criteria for copositive matrices, Linear Algebra Appl. 119 (1989), pp. 163-182. 
[39] S. Vempala. The Random Projection Method. Dimacs Series in Discrete Math., Vol 65, Amercian Mathematical Society, 2004.

[40] E.P. Wigner. On the distribution of the roots of certain symmetric matrices. Annals Math., 67:325-327, 1958.

[41] S. Yang and X. Li, Algorithms for determining the copositivity of a given matrix, Linear Algebra Appl., 430(2009), pp. 609-618. 